\title{
Model-Based Correction of Diffraction Effects of the Virtual Source Element
}

\author{
Erik Wennerström and Tadeusz Stepinski, Senior Member, IEEE
}

\begin{abstract}
A method for ultrasonic synthetic aperture imaging using finite-sized transducers is introduced that is based on a virtual source (VS) concept. In this setup, a focused transducer creates a VS element at its focal point that facilitates the use of synthetic aperture focusing technique (SAFT). It is shown that the performance of the VS method may be unsatisfactory due to the distortion introduced by the diffraction effects of the aperture used for creating the VS element. A solution to this problem is proposed that consists of replacing the classical SAFT by the extended synthetic aperature focusing technique (ESAFT) algorithm presented in our earlier works. In ESAFT, the full geometry of the VS is modeled, instead of applying the simplified point source approximation used when VS is combined with classical SAFT. The proposed method yields a substantial improvement in spatial resolution compared to that obtained using SAFT. Performance of the proposed algorithm is first demonstrated on simulated data, then verified on real data acquired with an array system.
\end{abstract}

\section{INTRODUCTION}

$\mathrm{C}$ Oonventional B-mode imaging, which is widely implemented in ultrasound array systems, suffers from the fact that lateral resolution depends on the depth in the image. The fixed focus principle used in such systems provides the best resolution only for the thin slice of the image containing echoes from the aperture's focal zone. This limitation can be quite severe in certain high-frequency applications in which only limited depth focal zones are obtained for relatively large apertures.

For slowly moving or stationary objects, the synthetic aperture focusing technique (SAFT) can be applied, which yields transmit and receive dynamic focusing with a uniform resolution [1], [2]. In practical implementations of SAFT imaging, there is a trade-off between the size of the transducer element and the requirement concerning signalto-noise ratio (SNR) in the received signal. Using a larger transducer element improves the SNR, but at the same time it reduces the quality of the reconstructed image because a large transducer introduces diffraction effects that remain uncompensated in classical SAFT schemes.

An elegant solution to this problem was presented by Passman and Ermert [3], who proposed a technique that involves treating the focal point of the transducer as a virtual source (VS) for synthetic aperture processing. In their formulation, distortion due to the diffraction effects of a

Manuscript received March 28, 2006; accepted July 26, 2006.

The authors are with Uppsala University, Signals and Systems Group, Uppsala, Sweden (e-mail: ew@signal.uu.se).

Digital Object Identifier 10.1109/TUFFC.2007.432 large transducer aperture are supposed to be minimized by forming the VS in the vicinity of the transducer's surface. A VS created by a focused transducer is assumed to produce approximately spherical waves in the region of interest (ROI) beyond the focal zone over a certain aperture angle. The virtual source technique (VST) has been investigated by Frazier and O'Brien [4], who examined its performance in terms of lateral resolution, sidelobe levels, spatial sampling rate, and SNR for images created at lower frequencies. An extension of the VST was proposed in [5] in the form of the all point transmit and receive focusing based on transmit synthetic focusing combined with receive dynamic focusing with a linear array transducer.

The results reported in this paper extend previous work concerning the VST by examining diffraction effects encountered when forming a VS. The result of this examination indicates that diffraction effects encountered in the vicinity of VS cannot be neglected because they impair the performance of classical SAFT algorithms that have been proposed for the VST. To solve this problem, we are proposing a new, improved version of the VST involving the extended synthetic aperture focusing technique (ESAFT) that was presented in our earlier work [6], [7]. We also compare the lateral resolution of the improved VST to that which can be obtained when the ESAFT is used directly to the aperture creating the VS.

The reminder of this paper is organized as follows: In Section III, the concept of VS is introduced. A theoretical background on spatial impulse responses is presented in Section IV. This background is needed for a further analysis of virtual sources, given in Section V. In Section VI, the imaging problem is reformulated as a reconstruction problem, and the ESAFT algorithm is presented. Section VII presents numerical results obtained from simulations and measurements. Conclusions are presented in Section VIII.

\section{Synthetic Aperture Imaging}

A common practice in sonar as well as in nondestructive evaluation (NDE) of materials is to coherently compound ultrasonic data from several different acquisitions, usually involving spatial diversity, to form images with enhanced lateral resolution. Typically, spatial diversity is achieved by moving either a transducer or an array aperture a short distance in a straight line between each acquisition. A postprocessing scheme then is applied to the data to achieve all point focusing in certain ROI. Spatial movement of the transducer (or the aperture) creates a synthetic aperture, 


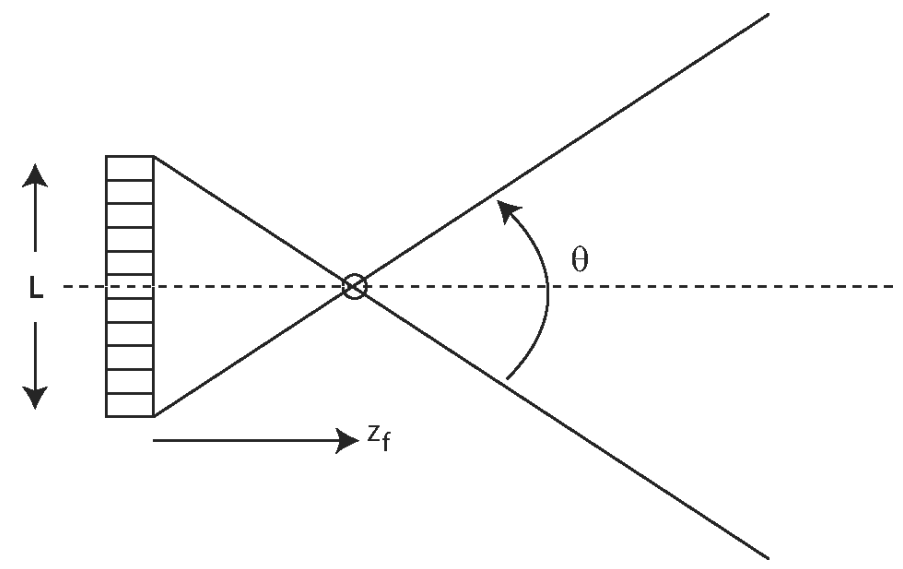

Fig. 1. A VS element in the transducers focal zone. The focal distance is $z_{f}$, the transducers size is $L$.

and the techniques involved in the imaging process are often referred to as synthetic aperture imaging (SAI). A number of algorithms have been proposed performing SAI both in time domain [8] and frequency domain [9].

SAFT, which is a widely used SAI method, achieves all point focusing in receive, either in time domain by delayand-sum operations or equivalently in frequency domain. SAFT assumes that the source transducer emits a spherical wave, transmitting all energy from a single point. It also assumes a diffraction-free, small, receiving transducer, so that the acoustic energy reflected from a scatterer arrives at the receiver in only one time instant. These assumptions are valid in farfield and require a small size transducer if the ROI is located close to the transducer. However, a small, active area also means low acoustic energy output, limiting ultrasound penetration into the inspected medium and decreasing the SNR.

\section{ViRTUAL SOURCES}

The concept of VS involves using a focused transducer to create a virtual source element located in the transducer's focal zone. The element is assumed to be a pointlike source of spherical waves within an aperture angle $\theta$, see Fig. 1. The beam emitted from the element is assumed to be approximated as an inverse of the beam before focal zone, and the aperture angle is defined by simple geometry as:

$$
\theta=2 \tan ^{-1} \frac{L}{2 z_{f}}
$$

where $L$ is the transducer width and $z_{f}$ is the focal distance [4]. If the spherical wave assumption is correct, this would be beneficial for the SAFT algorithm, as the assumptions on point-like transducer and spherical wave propagation would be met. To further investigate the properties of the waves emitted from a VS element in time domain, we will introduce the concept of spatial impulse responses.

\section{The Spatial Impulse Response}

If particle velocity at all points at the surface of a finitesized transducer takes the form of Dirac pulse, the pressure wave created at different observation points in space will take the form of finite-length pulses, depending on the position of the point and the geometry of the transducer. The respective pulse shapes are determined by the spatial impulse response (SIR) characteristic for each observation point $\mathbf{r}$.

The pressure $p(\mathbf{r}, t)$ can be expressed as a surface integral over transducer's transmitting active area $S_{t}[10]$. If we assume that the normal velocity $v_{n}(t)$ at the transducer surface is space independent, this integral can be written as [11]:

$$
p(\mathbf{r}, t)=\rho_{0} v_{n}(t) * \frac{\partial}{\partial t} \int_{S_{i}} \frac{\delta\left[t-\left(\left|\mathbf{r}-\mathbf{r}_{S_{t}}\right| / c\right)\right]}{2 \pi\left|\mathbf{r}-\mathbf{r}_{S_{t}}\right|} d S_{t}
$$

Using this integral, we can define the spatial impulse response related to the geometry of the transmit aperture, the transmit SIR $h_{t}(\mathbf{r}, t)$ :

$$
h_{t}(\mathbf{r}, t)=\int_{S_{t}} \frac{\delta\left[t-\left(\left|\mathbf{r}-\mathbf{r}_{S_{t}}\right| / c\right)\right]}{2 \pi\left|\mathbf{r}-\mathbf{r}_{S_{t}}\right|} d S_{t}
$$

so the pressure can be expressed as:

$$
p(\mathbf{r}, t)=\rho_{0} v_{n}(t) * \frac{\partial}{\partial t} h_{t}(\mathbf{r}, t) .
$$

Assume a point reflector with the elementary surface $s_{e}$ at the observation point $\mathbf{r}$. In the same way that the transmit SIR relates the geometry of the aperture to the emitted pressure at $\mathbf{r}$, the similarly defined receive SIR, $h_{r}(\mathbf{r}, t)$, relates the point reflector to the total incident pressure at the aperture. In many cases, the same aperture is used in transmit and receive, and thus for an isotropic medium $h_{t}(\mathbf{r}, t)=h_{r}(\mathbf{r}, t)$.

The transmit and receive SIR relates normal velocity on the transducer surface, $v_{n}(t)$, through the reflection from a point scatterer, to the total incident pressure back on the surface again, $\langle p\rangle(\mathbf{r}, t)$. To relate these quantities to the electric input and output signals, $y_{i}(t)$ and $y_{o}(t)$ we need the transducer's transmit and receive electrical impulse responses $h_{e t}(t)$ and $h_{e r}(t)[12]$ :

$$
\begin{aligned}
& v_{n}(t)=h_{e t}(t) * y_{i}(t), \\
& y_{o}(t)=h_{e r}(t) *\langle p\rangle(\mathbf{r}, t) .
\end{aligned}
$$

The whole pulse-echo system is summarized using this impulse response notation in Fig. 2 .

Now, if we define the double path SIR:

$$
h(\mathbf{r}, t)=h_{t}(\mathbf{r}, t) * h_{r}(\mathbf{r}, t),
$$

and the double path electrical impulse response (see [6] for details):

$$
h_{e}(\mathbf{r}, t)=\frac{\rho_{0}}{2 c} \frac{\partial^{2}}{\partial t^{2}} * h_{e t}(t) * h_{e r}(t)
$$




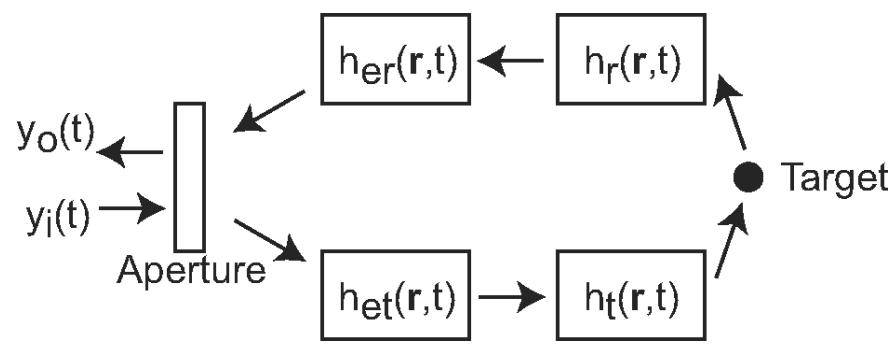

Fig. 2. Block diagram illustrating the convolution model pulse-echo measurements.

and combine those with (5), we get the complete expression of the imaging system from the electrical input signal to the received output signal:

$$
y_{o}(t)=s_{e} h(\mathbf{r}, t) * h_{e}(t) * y_{i}(t) .
$$

For simple geometries, such as a point or line, the SIR can be calculated analytically from (3), but for more complex geometries, the SIRs have to be calculated numerically. See [13] and for more details on calculating and sampling SIRs for different geometries. The double path electrical impulse response can be obtained from the measurements in a suitable setup.

\section{Properties of a Virtual Source}

General features of the VS were already characterized in Section III. The most important feature is the ability of the VS to emit spherical waves desired for the efficient implementation of SAFT. In this section, we present simulated results illustrating spatial characteristics of the acoustic field generated by a VS. All results are generated by the simulations of a thin strip transducer with a center frequency of $3 \mathrm{MHz}$. Both straight and concave (focused) strips were simulated. More details concerning the simulations can be found in Section VII-A.

\section{A. Axial Pressure of a Virtual Source Element}

Fig. 3 shows the maximum amplitude of the axial pressure as a function of distance for three different transducer geometries, two straight strips (4-mm and 32-mm long), and a $32-\mathrm{mm}$ concave strip. The concave strip forms a VS element with aperture angle $\theta=30^{\circ},(1)$. For a simple transducer geometry, such as a flat disc or line strip, the pressure amplitude in farfield should decrease inversely proportional to the distance $r$ [10]. For the VS obtained from the concave strip, the pressure amplitude, as expected, has a very distinct maximum in the focal zone. But in the region beyond the focal zone, it declines even more rapidly than $1 / r$. It is apparent that, at the distances where targets would be located, well beyond the virtual source element, the pressure amplitude is similar to that from much smaller, unfocused aperture, in this case only $4 \mathrm{~mm}$.

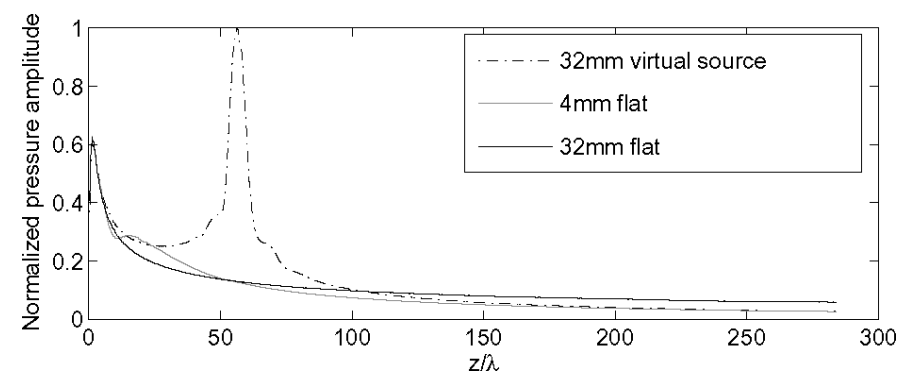

Fig. 3. Axial pressure amplitude for three simulated strip transducers.

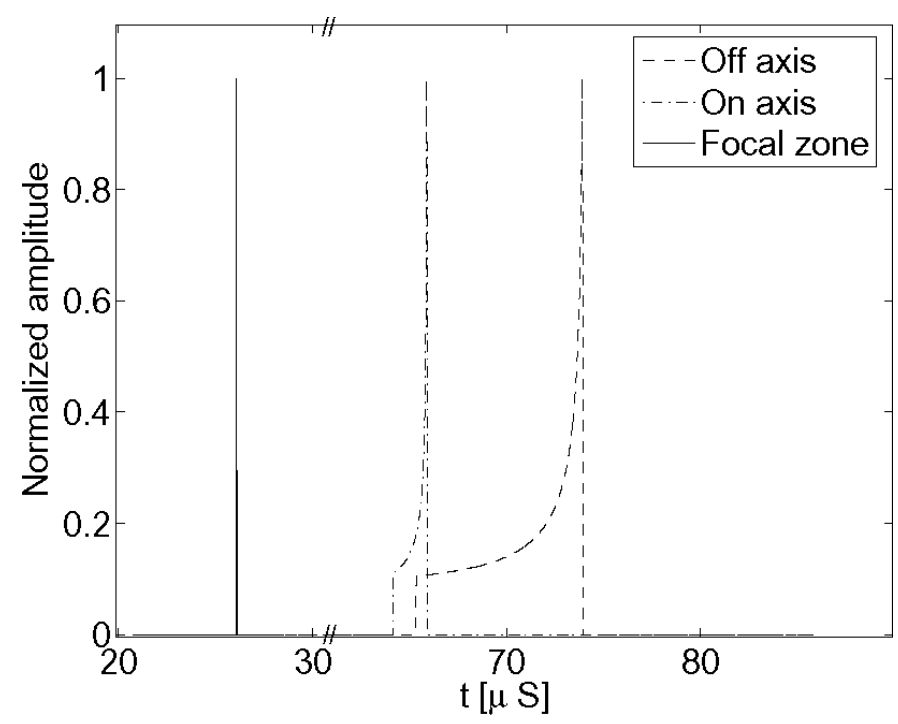

Fig. 4. Spatial impulse responses of three observation points for a focused transducer.

This property of the VS is highly undesired in terms of the penetration into medium and the resulting SNR. It is evident that the increased aperture size, which resulted in the desired divergent beam facilitating the use of the SAFT algorithm, did not yield the expected increase of pressure level in the ROI.

\section{B. Spatial Impulse Responses of a Virtual Source Element}

The assumptions in the standard SAFT algorithms about point-like transducer and spherical wave propagation is equivalent to assuming that the SIR for all observation points in the ROI is a delta-pulse. This is required by SAFT methods, as they compensate only for the different distances to observation points.

Example presented in Fig. 4 illustrates the shapes of SIRs of the simulated VS in different regions.

The VS was formed by a 32-mm strip transducer focused to a VS element with $30^{\circ}$ aperture angle. Please note that, for clarity, the electrical impulse response of the transducer elements are omitted. Three impulse responses presented in Fig. 4 were calculated for the following points: in the focal zone, beyond the focal zone (distance $3 z_{f}$ ), on the axis of the transducer, and off-axis at a distance of $3 z_{f}$. 


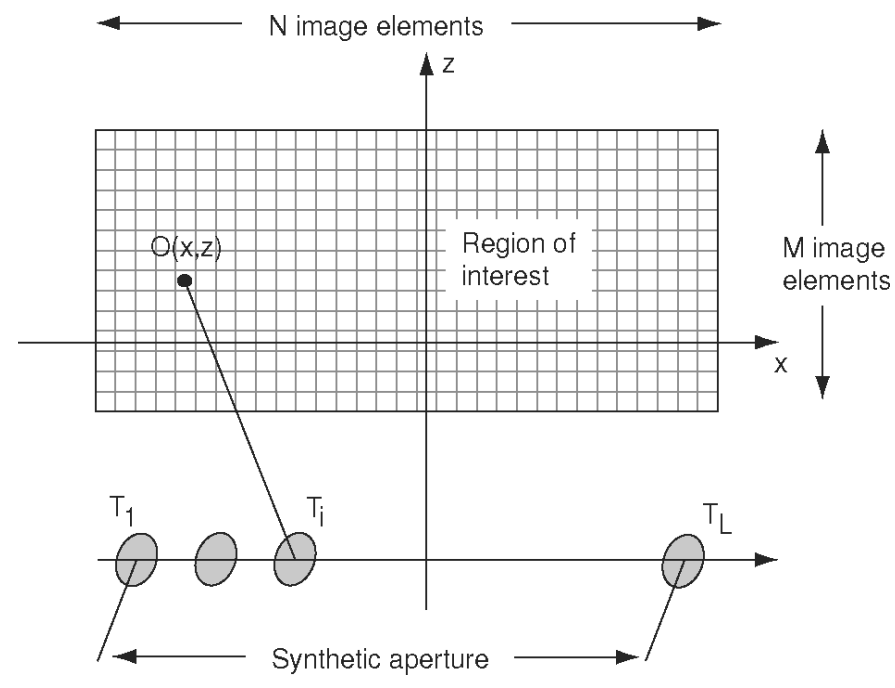

Fig. 5. Synthetic aperture data acquisition.

It is apparent that the SIR takes the delta-pulse form in only the focal point. In all other points the SIR has a considerable length, both on and off-axis. They are longer the further the observation point is from the focal zone and the further it is off the axis. Moreover, it is obvious that the pulse length will be proportional to the aperture size. In the most interesting region where the targets are expected, beyond the virtual source element, the deltapulse approximation cannot be applied. This means that the classical SAFT will perform poor in that region.

Summarizing the simulations, we have shown that there are two serious deficiencies of the VST related to the diffraction effect of a focused aperture. First, the pressure level beyond the focal zone will be much lower than expected. Second, well pronounced diffraction effects in that zone may impair SAFT performance. Relating to the example in the previous section, even though the wide strip focused to a VS element yields a much wider beam beyond the focal zone than the smaller strip, it suffers from very pronounced diffraction effects, making image reconstruction difficult. Despite the wider beam, there will not be a higher axial pressure at the ROI (Section V-A) nor better performance after delay-and-sum SAFT processing (Section VII-D).

\section{The THE ESAFT Algorithm}

The ESAFT algorithm, presented in our earlier work [6], is designed to compensate for the diffraction effects of an imaging system using the spatial impulse responses. The algorithm, which is based on a discrete matrix model of the imaging system results in a linear spatiotemporal filter.

\section{A. Two-Dimensional Model of an Imaging System}

In the monostatic SAI, data is acquired from a transducer operating in pulse-echo mode. The transducer is

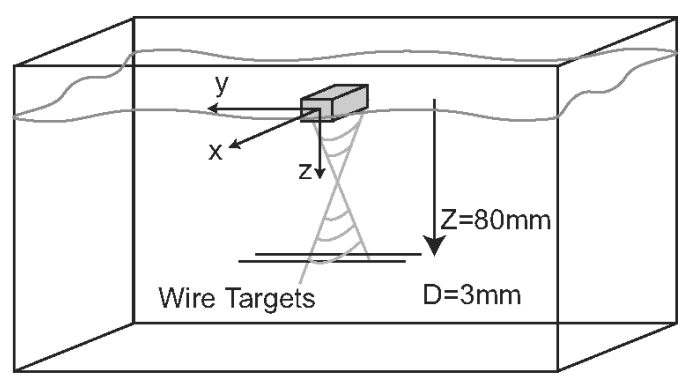

(a)

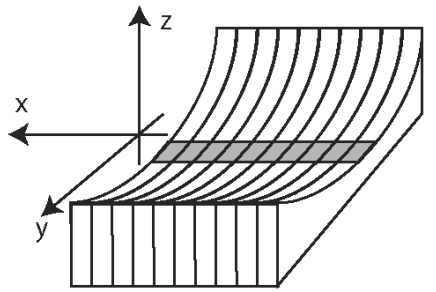

(b)

Fig. 6. (a) The measurement setup, wire targets in immersion. (b) The geometry of the array. At geometrical focus the array can be approximated with a thin strip (shaded).
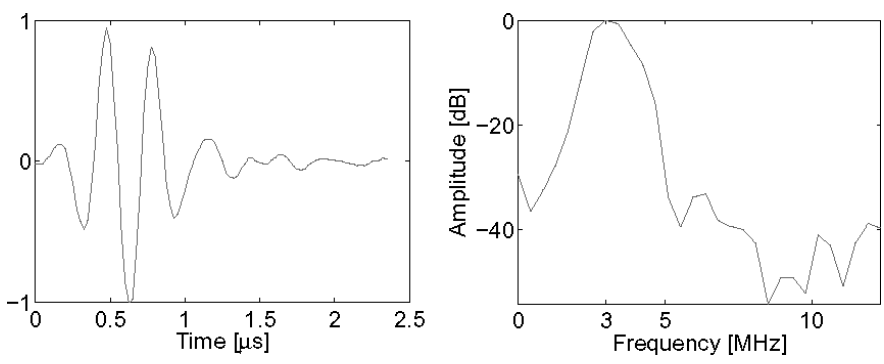

Fig. 7. Electric impulse response (left) of the transducer and its Fourier transform (right).

moved in space to acquire measurements at different positions in the setup shown in Fig. 5. The transducer is shifted in parallel to the $x$-axis, and the measurements are taken from $L$ positions. The ROI, denoted by $\mathbf{O}$, is divided into $M \times N$ image elements. The ROI is entirely in the $x z$-plane with $y=0$. Every element $o\left(x_{n}, z_{m}\right)$ is a scalar representing the scattering strength of a target in the position $\left(x_{n}, z_{m}\right)$. The measurement set consists of $L$ discrete time A-scans, each containing $K$ samples.

Let the $K \times 1$ vector $\mathbf{y}_{l}$ denote one such noise-free Ascan response at transducer position $T_{l}$. Using superposition, it can be expressed as a sum of echoes from all scatterers in the ROI:

$$
\begin{aligned}
\mathbf{y}_{l} & =\sum_{n=0}^{N-1} \sum_{m=0}^{M-1} \mathbf{h}\left(T_{l}, x_{n}, z_{m}\right) * \mathbf{h}_{e} * \mathbf{y}_{i} o\left(x_{n}, z_{m}\right) \\
& =\sum_{n=0}^{N-1} \sum_{m=0}^{M-1} \mathbf{p}_{l}\left(x_{n}, x_{m}\right) o\left(x_{n}, z_{m}\right)
\end{aligned}
$$

where $\mathbf{h}\left(T_{l}, x_{n}, z_{m}\right)$ is the double path SIR from the point at $T_{l}$ to $o\left(x_{n}, z_{m}\right)$, see (6). Using this notation, it is possible to form the $K \times M$ matrices: 

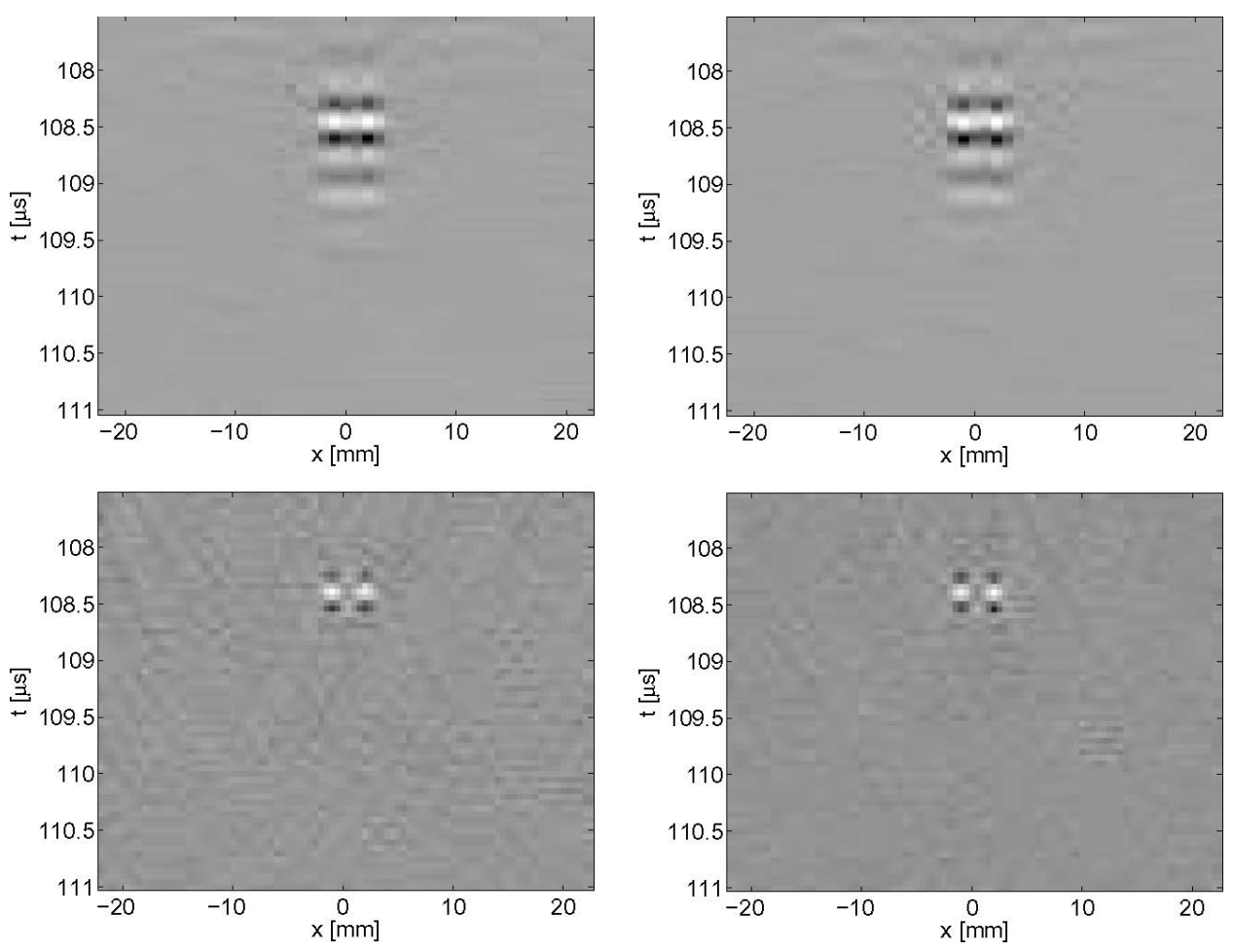

Fig. 8. Simulation results for the 4-mm transducer. Standard SAFT, without VS (top left), standard SAFT, using VS (top right). ESAFT, without VS (bottom left), and ESAFT using VS (bottom right).

$$
\mathbf{P}_{l}(n)=\left(\mathbf{p}_{l}\left(x_{n}, z_{1}\right) \ldots \mathbf{p}_{l}\left(x_{n}, z_{M}\right)\right),
$$

for all combinations of transducer positions $l$ and columns in $\mathbf{O}$. Now the SIR matrix $\mathbf{P}$ can be constructed as:

$$
\mathbf{P}=\left(\begin{array}{cccc}
\mathbf{P}_{0}(0) & \mathbf{P}_{0}(1) & \ldots & \mathbf{P}_{0}(N-1) \\
\mathbf{P}_{1}(0) & \mathbf{P}_{1}(1) & \ldots & \mathbf{P}_{1}(N-1) \\
\vdots & & & \vdots \\
\mathbf{P}_{L-1}(0) & \mathbf{P}_{L-1}(1) & \ldots & \mathbf{P}_{L-1}(N-1)
\end{array}\right)
$$

After stacking both the data $\mathbf{y}$ and the image $\mathbf{o}$ into column vectors, the model of the imaging system can be expressed in this compact matrix notation:

$$
\mathbf{y}=\mathbf{P o}+\mathbf{e},
$$

where $\mathbf{e}$ is a vector with additive noise.

It is worth noting that a similar model can be developed for multistatic SAI, it is only a matter of reorganizing the SIR matrix $\mathbf{P}$. The ESAFT method described in the next section is applicable to the multistatic case as well. In this work, measurements were performed using the monostatic approach, due to limitations of the available acquisition equipment.

\section{B. Image Reconstruction}

Focusing in the ESAFT algorithm is formulated as a linear reconstruction problem.

Define the reconstruction filter $\mathbf{K}$ so that the estimate of the reconstructed image $\hat{\mathbf{o}}$ :

$$
\hat{\mathbf{o}}=\mathbf{K y},
$$

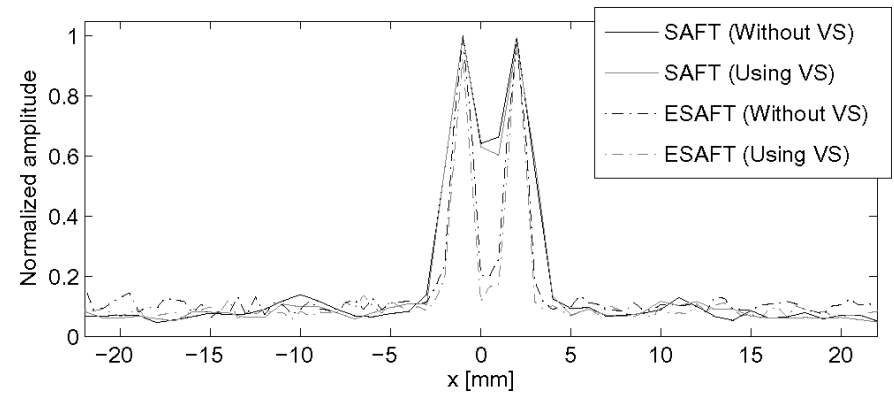

Fig. 9. Profile plot obtained from the simulation of the 4-mm aperture. Amplitude is normalized to unity maximum.

minimizes the reconstruction error $(\mathbf{o}-\hat{\mathbf{o}})$ in the mean square error sense, that is:

$$
\mathbf{K}=\arg \min _{\mathbf{K}} E\left\{\|\mathbf{o}-\hat{\mathbf{o}}\|^{2}\right\}=\arg \min _{\mathbf{K}} E\left\{\|\mathbf{o}-\mathbf{K y}\|^{2}\right\} .
$$

If both $\mathbf{o}$ and $\mathbf{e}$ are assumed to be Gaussian, mutually uncorrelated random vectors with covariance matrices $\mathbf{C}_{o}$ and $\mathbf{C}_{e}$, respectively, (15) takes the form:

$$
\begin{aligned}
\mathbf{K} & =\arg \min _{\mathbf{K}} E\left\{\|\mathbf{o}-\mathbf{K}(\mathbf{P o}+\mathbf{e})\|^{2}\right\} \\
& =\arg \min _{\mathbf{K}} \operatorname{tr}\left(\mathbf{C}_{o}\right)-2 \operatorname{tr}\left(\mathbf{K}^{T} \mathbf{C}_{o} \mathbf{P}^{T}\right) \\
& +2 \operatorname{tr}\left(\mathbf{K} \mathbf{P} \mathbf{C}_{o} \mathbf{P}^{T} \mathbf{K}^{T}\right)+\operatorname{tr}\left(\mathbf{K} \mathbf{C}_{e} \mathbf{K}^{T}\right) .
\end{aligned}
$$

Taking the derivative of (16) and equating it to zero yields the reconstruction filter [12]:

$$
\mathbf{K}=\mathbf{C}_{o} \mathbf{P}^{T}\left(\mathbf{P C}_{o} \mathbf{P}^{T}+\mathbf{C}_{e}\right)^{-1} \mathbf{y} .
$$



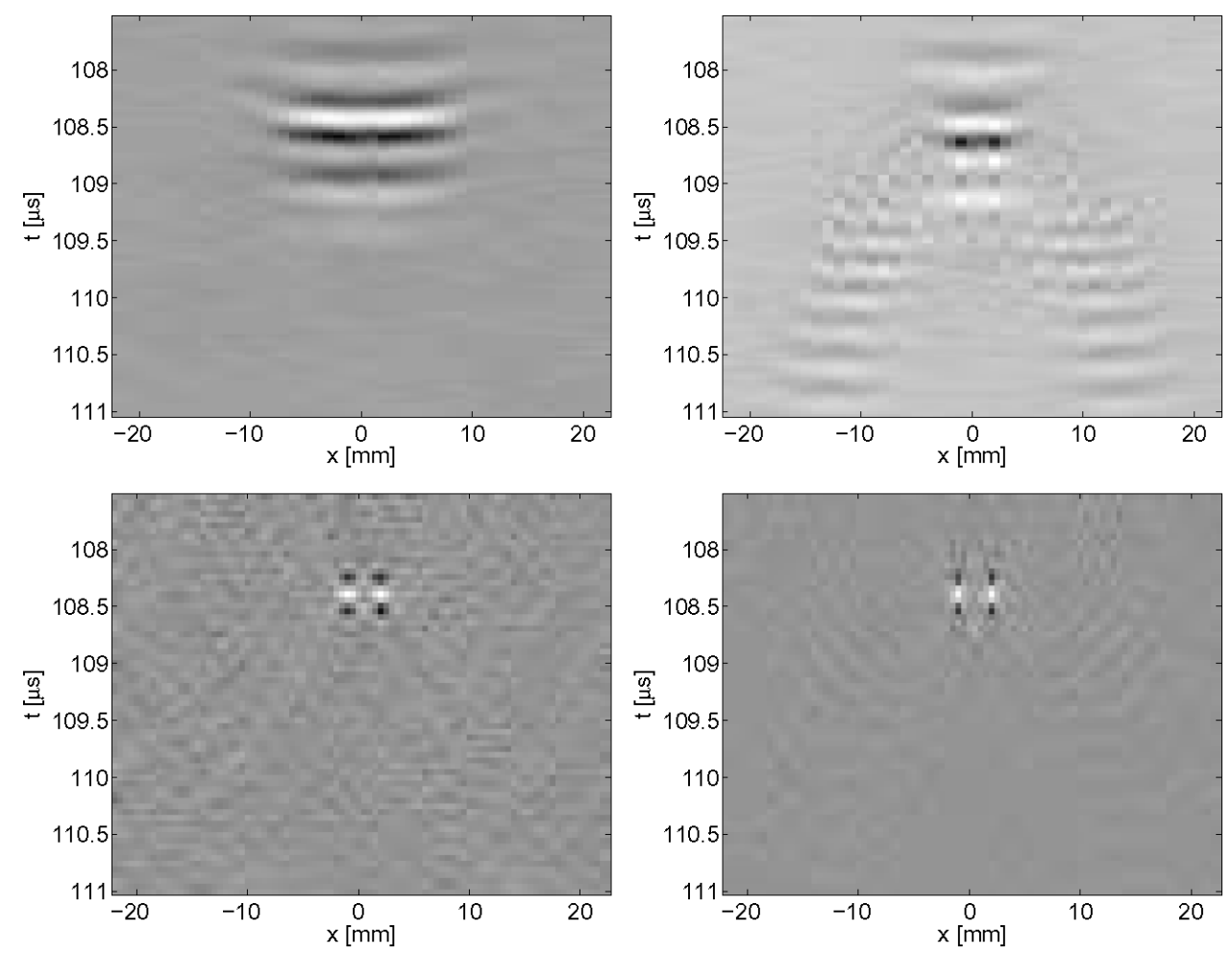

Fig. 10. Simulation results for the 16-mm transducer. Standard SAFT, without VS (top left), standard SAFT, using VS (top right). ESAFT, without VS (bottom left) and ESAFT using VS (bottom right).

The main advantage of this method is its ability to reconstruct images acquired using any transducer geometry provided that the respective SIRs included in the matrix $\mathbf{P}$ are known. ESAFT yields good performance even for larger apertures. The results and comparison presented in Section VII-D can serve as a good illustration of this property.

In the presentation of the ESAFT algorithm in the previous sections, there are two requirements for the method to be applicable: scatterers need to be small and the medium they are located in needs to be homogenous. Well defined boundaries between different media, such as those encountered in the inspection of a solid in immersion, do not pose a problem. See [12] for more details of calculating SIRs for observation points in an immersed solid.

\section{RESUltS}

\section{A. Experimental Setup}

Experiments and simulations were performed to evaluate the effect of using VS combined both with time domain delay-and-sum SAFT and with ESAFT. A linear rectangular array with center frequency of $3 \mathrm{MHz}$ was used in the experiments. The array consisted of 32 elements with width $1 \mathrm{~mm}$ that were bridged to form a variable sized aperture. Each array element was geometrically focused in elevation ( $y$-direction in Fig. 5 and Fig. 6) at a depth of $80 \mathrm{~mm}$. As long as targets only are present at this distance range and the ROI is in the $x z$-plane $(y=0)$, the array

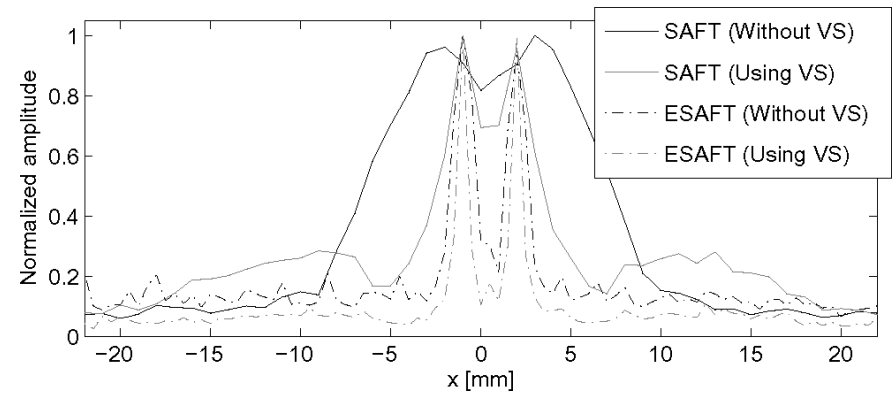

Fig. 11. Profile plot obtained from the simulation of the 16-mm aperture. Amplitude is normalized to unity maximum.

can be approximated as a thin strip. The array's double path electrical impulse response and its Fourier transform are shown in Fig. 7.

The shortest aperture used in experiments consisted of 4 elements, and the longest included 16 elements. The aperture lengths were determined by the limitations of the acquisition system in focusing mode. The elements in the focused apertures were excited with pulses delayed to obtain focusing in the $x$-direction needed to form the VS elements.

Two steel wires with diameter $0.3 \mathrm{~mm}$, separated by a distance $D=3 \mathrm{~mm}$, were used as targets. The targets were immersed in water at a distance of $80 \mathrm{~mm}$ from the array. The measurements and simulations were performed both for unfocused apertures and for the apertures focused to form a VS element at $28 \mathrm{~mm}$. Data was acquired in a B-scan mode, as described in Section VI-A. The trans- 

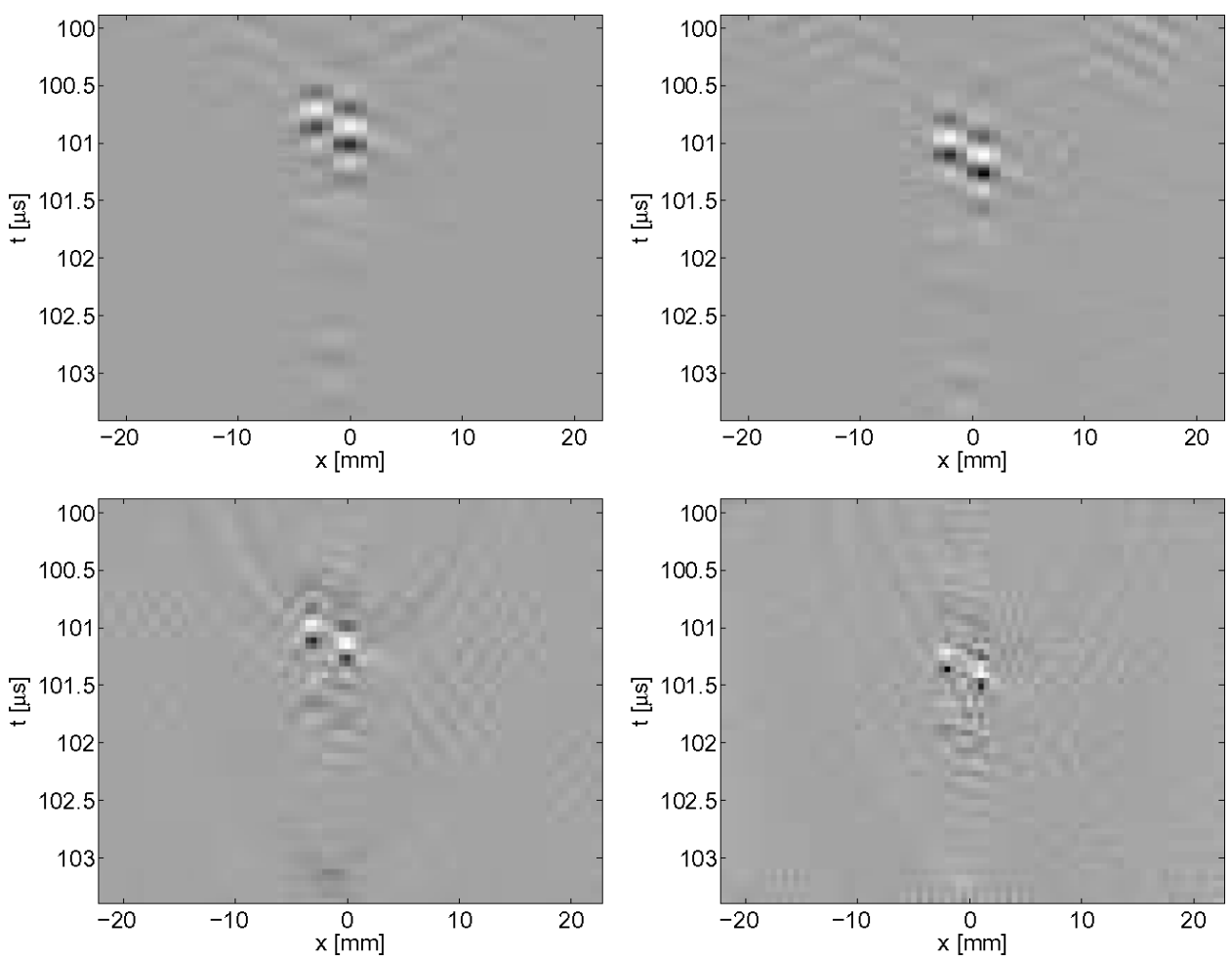

Fig. 12. Results from measurements for the 4-mm transducer. Standard SAFT, without VS (top left), standard SAFT, using VS (top right). ESAFT, without VS (bottom left) and ESAFT using VS (bottom right).

ducer was shifted along the $x$-axis so that the wire targets were perpendicular to the motion. The spatial sampling distance used was $1 \mathrm{~mm}$, equal to twice the wavelength of the ultrasound in water. Ultrasonic signals were sampled at $40 \mathrm{MHz}$ and converted using an 8-bit analog-to-digital converter.

Simulated and measured data was processed with standard delay-and-sum SAFT. Linear interpolation between samples was used in the SAFT implementation. No apodization was applied to the synthetic aperture. The synthetic aperture consisted of 24 measurements for most of the data sets, but it was allowed to vary to find the best possible performance of the method. Measured and simulated images also were reconstructed using the ESAFT method according to (14) and (17).

\section{B. Simulations}

Simulations were done for a measurement setup identical to that described in the previous section. All simulations were done using the DREAM (Discrete REpresentation Array Modeling) toolbox [13]. The combined electrical impulse responses and excitation pulse (see Section IV) used in the simulations were measured from the real system.

The results are displayed in Figs. 8 to 11 in which results of simulating the following four different configurations are presented: standard SAFT used with an unfocused real aperture, standard SAFT with a VS element, ESAFT used for processing of an unfocused real aperture, and ESAFT used with a VS element. The intensity images represent

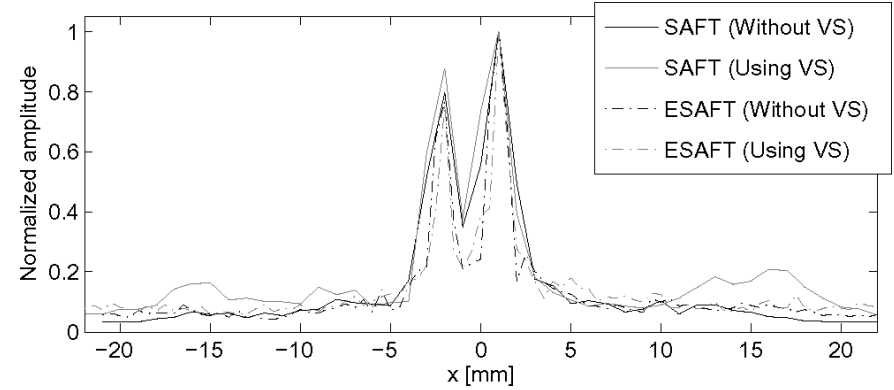

Fig. 13. Profile plot obtained from measurements with the 4-mm aperture. Amplitude is normalized to unity maximum.

amplitudes of the RF signals of the result of processing for these four configurations. The profile plots in Figs. 9 and 11 are composed of the maximum value from each column ( $x$ position) in the intensity images. Each of the profiles are normalized to have a maximum amplitude of one, to enable comparison of profiles from different algorithms.

Results presented in Figs. 8 to 11 illustrate the problem indicated in Section V-the VST does not perform well due to the diffraction effects of a large aperture creating the VS. For the small aperture $(4 \mathrm{~mm})$, the impact of focusing is not very apparent. This is due to two reasons. First, the approximation with a point source is quite realistic. Second, focusing is rather inexact with only four array elements. For the larger aperture $(16 \mathrm{~mm})$, however, the difference is much more notable. The point source assumption is not valid any longer for the planar aperture, making the standard SAFT algorithm inefficient. With focusing, 

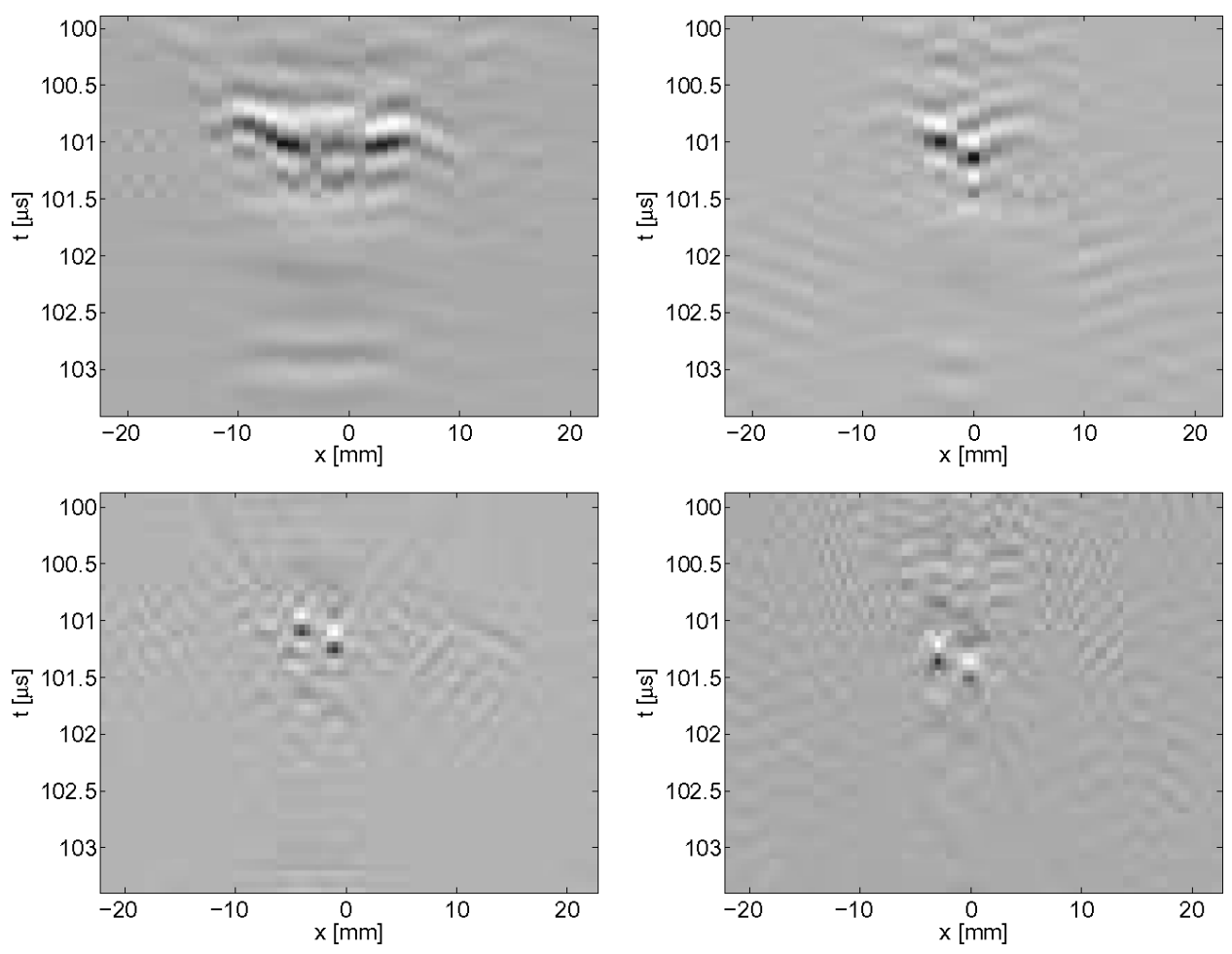

Fig. 14. Results from measurements for the 16-mm transducer. Standard SAFT, without VS (top left), standard SAFT, using VS (top right). ESAFT, without VS (bottom left) and ESAFT using VS (bottom right).

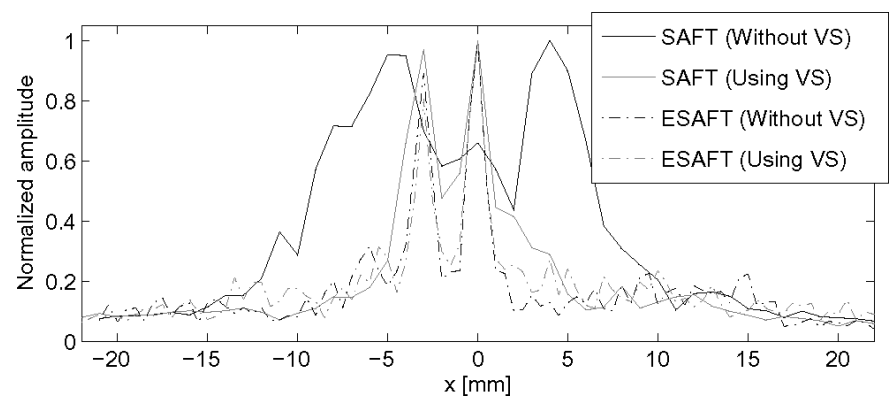

Fig. 15. Profile plot obtained from measurements with the 16-mm aperture. Amplitude is normalized to unity maximum.

however, the two targets can be distinguished easily in both Figs. 10 and 11. The model-based ESAFT method performs well both for the plane and focused real aperture. It accurately compensates the diffraction effects, and both targets can be identified easily. The result obtained with a VS and ESAFT is supreme in terms of resolution (sharper peaks) and the level of background noise.

\section{Measurements}

Measurements were performed using the setup and the array described in Section VII-A. The RF signals were sampled at $40 \mathrm{MHz}$ with an 8-bit A/D converter. The results from the measurements and postprocessing are shown in Figs. 12 to 15.

The measurement results are in good agreement with the simulations. For the small aperture, there is small or

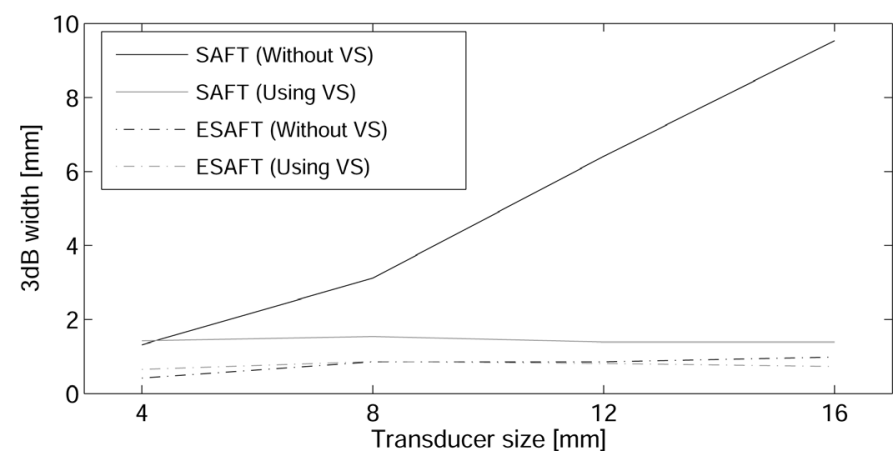

Fig. 16. Resolution of the tested methods as a function of aperture size.

no benefit from the VS approach. For a larger transducer, the use of a VS element substantially improves the results yielded by the SAFT algorithm. The model-based ESAFT algorithm is affected only slightly by the size of the transducer, as it models the complete transducer geometry, without the point source approximation used in SAFT.

\section{Impact of Transducer Size}

To evaluate lateral resolution of all tested methods, similar measurements were repeated for a single target. The $3 \mathrm{~dB}$ width $x_{3 d B}$ of the single target profile is used as the resolution measure. In Fig. 16 the $x_{3 d B}$ width for SAFT and ESAFT, with and without focusing to a VS element, is plotted as a function of transducer size. 
It is evident that the VST can yield a substantial performance improvement for the SAFT algorithm. The ESAFT algorithm offers a higher resolution for any transducer size, focused to a VS or not, as the method compensates for the transducer geometry. The SAFT algorithm used without the VST offers a resolution about half the transducer size, a result that is in agreement with theory [2].

It appears that the larger apertures actually provide a better resolution for ESAFT than the smaller. A larger aperture in this measurement also means a larger aperture angle for the VS, as the distance to the focal zone is the same. The wider beam provides more angular information in the B-scan, as the target is illuminated by a larger number of successive scans. The ESAFT algorithm is able to use this extra information for the image reconstruction, hence the improved resolution.

\section{CONCLUSIONS}

The use of the VST for synthetic aperture imaging with classical time-domain SAFT algorithm and the recently proposed ESAFT algorithm have been studied. In Section $\mathrm{V}$ it was shown that the acoustic field emitted from a VS element may take the form far from the spherical wave. Neither does the echo reflected from a scatterer located in the ROI beyond the focal zone take the form of a delta-pulse.

Simulations and measurements have confirmed that, although the use of the VST yields a substantial increase in the performance of the SAFT algorithm, the resulting lateral resolution still may be unsatisfactory. Further improvement in the resolution can be obtained by replacing the classical SAFT with ESAFT that is capable of compensating for the diffraction effects produced by realistic VS elements.

Results of the simulations and measurements have shown that processing the ultrasonic data obtained from a VS setup with ESAFT yields superior results in terms of both lateral resolution and background noise level. However, similar performance can be obtained even when ESAFT is directly applied to the array aperture used for creating the VS.

\section{REFERENCES}

[1] S. Norton, "Synthetic aperture imaging with arrays of arbitrary shape-Part I: General case," IEEE Trans. Ultrason., Ferroelect., Freq. Contr., vol. 49, pp. 399-403, Apr. 2002.

[2] L. Cutrona, "Comparison of sonar system performance achievable using synthetic-aperture technique with the performance achievable by more conventional means," J. Acoust. Soc. Amer., vol. 58, no. 2, pp. 336-348, Aug. 1975.

[3] C. Passman and H. Ermert, "A $100 \mathrm{MHz}$ ultrasound imaging system for dermatologic and ophthalmologic diagnostics," IEEE Trans. Ultrason., Ferroelect., Freq. Contr., vol. 43, no. 4, pp. 545-552, July 1996.
[4] C. Frazier and W. D. O'Brien, Jr., "Synthetic aperture techniques with a virtual source element," IEEE Trans. Ultrason., Ferroelect., Freq. Contr., vol. 45, pp. 196-207, Jan. 1998.

[5] M.-H. Bae and M.-K. Jeong, "A study of synthetic-aperture imaging with virtual source elements in B-mode ultrasound imaging systems," IEEE Trans. Ultrason., Ferroelect., Freq. Contr., vol. 47, pp. 1510-1519, Nov. 2000.

[6] F. Lingvall, T. Olofsson, and T. Stepinski, "Synthetic aperture imaging using sources with finite aperture: Deconvolution of the spatial impulse response," J. Acoust. Soc. Amer., vol. 114, no. 1, pp. 225-234, July 2003.

[7] F. Lingvall, "A method of improving overall resolution in ultrasonic array imaging using spatio-temporal deconvolution," $U l$ trasonics, vol. 42, pp. 961-968, 2004.

[8] M. Karaman, P. Li, and M. O'Donnell, "Synthetic aperture imaging for small scale systems," IEEE Trans. Ultrason., Ferroelect., Freq. Contr., vol. 42, no. 3, pp. 429-442, May 1995.

[9] M. Soumekh, Synthetic Aperture Radar Signal Processing with MATLAB Algorithms. 1st ed. New York: Wiley, 1999.

[10] L. Kinsler, A. Frey, A. Coppens, and J. Sanders, Fundamentals of Acoustics. 4th ed. New York: Wiley, 2000.

[11] A. Lhéemery, "Impulse-response method to predict echoresponses from targets of complex geometry. Part I: Theory," J. Acoust. Soc. Amer., vol. 90, pp. 2799-2807, Nov. 1991.

[12] F. Lingvall, "Time domain reconstruction methods for ultrasonic array imaging," Ph.D. dissertation, Signals and Systems, Uppsala University, Uppsala, Sweden, 2004.

[13] B. Piwakowski and K. Sbai, "A new approach to calculate the field radiated from arbitrarily structured transducer arrays," IEEE Trans. Ultrason., Ferroelect., Freq. Contr., vol. 46, pp. 422-440, 1999.

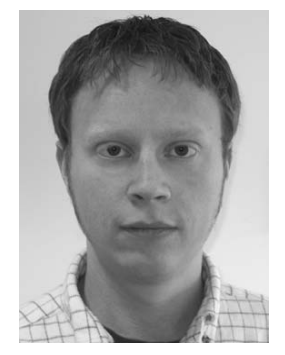

Erik Wennerström was born in 1977 in Sweden. He received an M.Sc. degree in engineering from Uppsala University, Uppsala, Sweden. He has been a Ph.D. student at the Signals and Systems group at Uppsala University since 2003 .

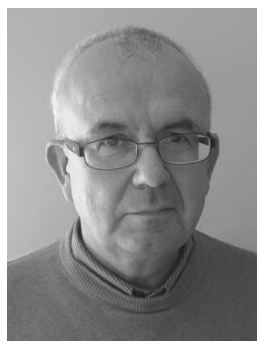

Tadeusz Stepinski was born in December, 1950 in Poland. He obtained an M.Sc. degree in electrical engineering from the Technical University of Szczecin, and a Ph.D. degree from the Technical University of Warsaw, Poland in 1973, and 1983, respectively. Since 1988 he has been with Signals and Systems group at Uppsala University, Uppsala, Sweden.

His research has been concerned with the application of modern signal processing to nondestructive evaluation of materials (NDE). His research interests are in the areas of measurement engineering and signal processing with focus on ultrasound, array processing and transducer design. He also has served as a lecturer teaching courses for the undergraduate students on the subjects of signal processing, NDE, virtual measurement systems, and sensors.

In 2002 he was appointed full Professor in Measurement Engineering at Uppsala University. He is author and co-author of more than 100 journal and conference papers. Dr. Stepinski is a senior member of IEEE, mand a member of the Acoustical Society of America, the American Society for NDT, and the British Institute of NonDestructive Testing. He chairs the Committee for New Techniques and Development in FOP (Swedish Society for NDT). 